\title{
Designing for Expert Users on a Large Non-standard Display
}

Henri Palleis

henri.palleis@ifi.Imu.de

\section{Fabian Hennecke}

fabian.hennecke@ifi.Imu.de

\section{Axel Hösl}

axel.hoesl@gmx.de

Heinrich Hussmann

hussmann@ifi.Imu.de

University of Munich (LMU)

Media Informatics Group

Amalienstr. 17

80333 München

Germany

Permission to make digital or hard copies of part or all of this work for personal or classroom use is granted without fee provided that copies are not made or

distributed for profit or commercial advantage and that copies bear this notice and the full citation on the first page. Copyrights for third-party components this work must be honored. For all other uses, contact the Owner/Author.

Copyright is held by the owner/author(s)

ITS'13, October 6-9, 2013, St. Andrews, United Kingdom.

ACM 978-1-4503-2271-3/13/10.

http://dx.doi.org/10.1145/2512349.2514915

\begin{abstract}
Testing new interface concepts for expert users on large and immobile display prototypes complicates the application of a user-centered design approach. In this paper we report on our experience with developing an emergency management system on a large curved display using an iterative user-centered design process. Involving the expert users was a major challenge due to the immobility of our display prototype. We present and discuss different prototyping and evaluation strategies and assess their suitability for such a scenario.
\end{abstract}

\section{Author Keywords}

Curved Display; Interactive Surfaces; User-centered Design Approach

\section{ACM Classification Keywords}

H.5.m. Information interfaces and presentation (e.g. $\mathrm{HCI}$ ): Miscellaneous.

\section{INTRODUCTION}

Large interactive displays with special form factors started to draw growing interest within the $\mathrm{HCI}$ community $[3,4]$. Two examples for this interest are Curve [7] and BendDesk [5], which propose to combine the distinctive advantages of horizontal and vertical workspaces through a continuous curved connection. 


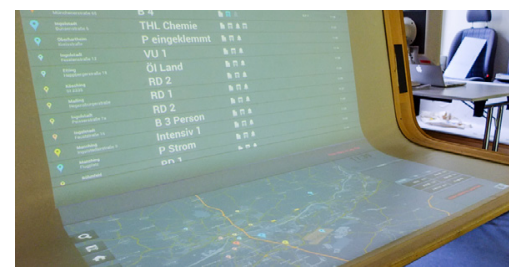

Figure 1. Our curved display prototype displaying the elaborated high fidelity prototype of the emergency management application.

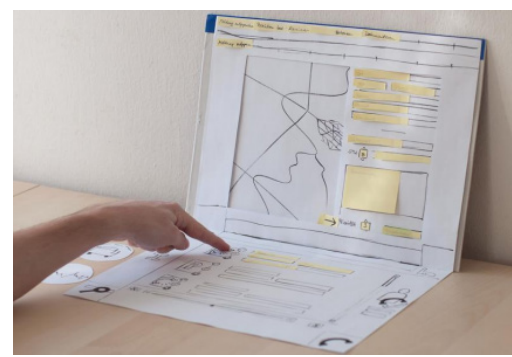

Figure 2. Small scale paper prototype consisting of two DIN A3 cardboards with paper add-ons
Designing interfaces for such large interactive surfaces in a user-centered design (UCD) process for expert users, however, can be challenging. Moving the display often implies the deconstruction, reassembly and recalibration of the system. The other way around, requiring the expert users to visit the lab to use the prototype can prove difficult due to their limited time. Additionally, the special form factors of such devices complicate the realistic simulation of direct interactions with paper prototypes.

We faced these challenges when we collaborated with expert users in a remote state-run emergency control center to create a novel user interface for coordinating emergencies for our curved display prototype (figure 1). There, agents work on multi-monitor setups and their typical workflow consists of answering an emergency call, collecting data, scheduling the rescue services and documenting the operation. Solving known usability problems of multi-monitor setups (bezel problems, window management, cursor loss, and distal information access [2]) promise to have a positive impact on performance and stress-resistance. Neither being able to bring our display prototype to their control center nor to regularly request the expert users to visit our lab, in the beginning we were not sure if a UCD process was applicable at all.

In this paper, we present different modifications of existing design methods and how we used them for shaping the user interface concept in an iterative and user-centered manner.

\section{THE DESIGN PROCESS}

Our UCD process consisted of four iterative phases: in situ expert interviews, paper prototyping, ergonomic optimization and high-fidelity prototyping. Some of the expert users were involved in several interview and prototyping sessions, while others only attended once, which helped us to collect feedback from both continuous and one-time usage.

A first interview session with two agents from the control room was conducted in order to get a sense of the current workflow and to introduce the interactive curved display. We explained the idea behind the interface concept using pictures of the actual prototype and got some initial ideas on how to adjust their current user interface elements to fit on our curved display, such as using a large interactive map on the horizontal part of the display as a central user interface element.

We then developed a small-scale paper prototype consisting of two DIN A3 paper sheets (figure 2) and defined an emergency call task to evaluate the prototype. This task was based upon the information gathered in phase one and refined in terms of number and order of sub-tasks during the entire UCD process. The first paper prototype was evaluated by six controllers and additionally by two interaction designers. Before the controllers executed the task, we described the concept of our interactive curved display once more to ensure a basic understanding of the type of device and its actual proportions.

Presenting the small-scale paper prototype worked well for verifying our understanding of the workflow and the comprehensiveness of the prototyped features. This resembles findings from media facade prototyping [6]. But it also caused some problems: doubtful, skeptic as well as amused reactions by the expert users, which 


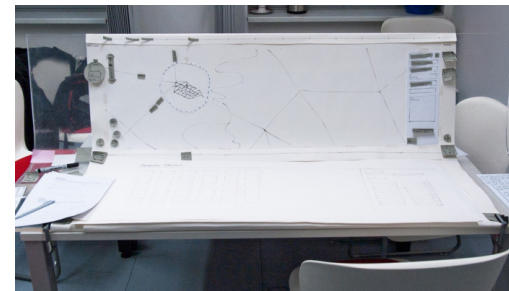

Figure 3. Full-scale paper prototype with appropriate paper add-ons.

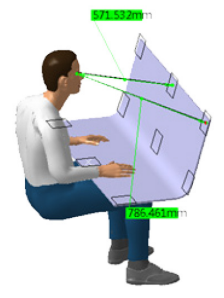

Figure 4. RAMSIS human mode sitting behind a 3D model of the curved display. might be explained by the lack of experience with prototyping methods, the discrepancy between daily occupation with potentially fatal emergencies in a legally highly regulated work environment and the look and feel of rather small sheets of paper. Moreover, the small-scale paper prototype could not convey a sense of the real device's size and proportions and did not have a curved connection.

Therefore we built a full-scale paper prototype (figure 3). A model of the display's shape made of acrylic glass was used as a base for the paper sheets $(125 \mathrm{~cm} x$ $85 \mathrm{~cm}$ ). The prototype was transported to the control room and evaluated with three controllers. They executed the emergency call task again and provided feedback in a semi-structured interview afterwards.

The full-scale paper prototype gave the controllers a more realistic impression of the actual display and led to higher engagement in the task and active ideation. However, changing the large paper screens during the tasks was unhandy, time-consuming and disrupting. For paper prototypes of large dimensions we therefore propose to rather conduct participatory design sessions, where users can freely explore the display and associate ideas based on a given prototype instead of strictly walking through tasks with many interface changes.

In further interviews we elaborated and validated a comprehensive sequence diagram formalizing the process of operating an emergency call. It should help us during the final interface design. To assess ergonomic aspects of the user interface design, we used the RAMSIS model [1], which has been developed to support the construction of ergonomic workspaces (figure 4). The results of the analysis provided information about reachability as well as minimum font size and icon spacing based on the regions of the display depending on the size of the human model.

We then sketched storyboards based on the sequence diagram to build a consistent representation of all states of the system. The storyboards were evaluated informally with an interaction designer. On the basis of these hand-drawn sketches, high-fidelity screens were designed with a graphics editing software (figure 5). Scaling up to the full size of the actual display required redefining sizes of interface elements from the handmade drawings. The results from the ergonomic analysis were used to assure readability as well as reachability for these elements.

The evaluation of the high-fidelity screens was carried out in two separate parts. In a first study, five HCI experts were involved in a role-play situation. We asked them to observe us developers operating the system while acting as actual controllers based on our task descriptions from the third phase. To create a more immersive experience, we used an unobtrusive foot pedal to trigger screen transitions when touching the display. This method helped the observers to focus on the interactions and processes, suggesting that it is a simple and effective method to simulate interactivity for defined task-based tests without an actual interactive prototype. Beyond that, it can be helpful to create digital low-fidelity prototypes in situation where paper prototypes become unmanageable.

In a second study, system walkthroughs were conducted with three controllers at the control center. Since the display could not be transported, the 


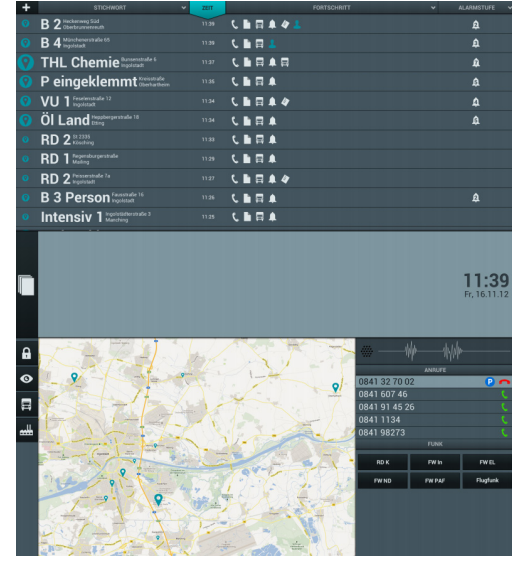

Figure 5. Elaborated high-fidelity screen. application screens were projected on a wall. During the session we zoomed in on the relevant regions of the interface to assure a good readability despite the projection. This study enabled us to check the general manageability of the workflow using our display and the interface. But one has to keep in mind that the wallbased projection made all content of the screens visible at once, whereas the actual display configuration implies a different visibility on the different display areas.

\section{CONCLUSION AND FUTURE WORK}

In this paper we have reported on our experiences with developing an expert application based on a curved display. We applied a UCD process that bypasses some of the arising challenges by slightly modifying existing prototyping methods. In particular, we distinguished between a simplified small-scale prototype and a transportable full-scale prototype. A small-scale prototype is a convenient tool to quickly test basic functionality, but can fail to communicate important features of the actual display such as special form factors and proportions. Therefore, a transportable fullscale prototype should be used to convey a sense for the real dimensions of the display. Complementing the UCD with an ergonomic analysis provides evidence on readability and reachability, which are important parameters when designing high-fidelity mockups. Based on our experience we recommend integrating such early high-fidelity screen designs into the development process. For future projects, we are interested in experimenting more with digital prototyping. For instance, using a pair of tablets or dual-screen devices might reduce the connotations of amusement and ridiculousness as experienced in the first phase. Similarly, instead of projecting flat highfidelity screens on a wall, a set of projectors could be used to create a more realistic impression of the actual display shape during the design process in terms of visibility and field of view.

\section{Acknowledgements}

We thank Thomas Gläser and Fabian Günzkofer for their support.

\section{REFERENCES}

\section{[1] RAMSIS,}

http://www.Ife.mw.tum.de/de/research/labs/ramsis.

[2] Robertson, G., Czerwinski, M., Baudisch, P., Meyers, B., Robbins, D., Smith, G. and Tan, D. The Large-Display User Experience. IEEE Comput. Graph. Appl. 25, 4 (July 2005), 44-51.

[3] Rümelin, S., Beyer, G., Hennecke, F., Tabard, A. and Butz, A. Towards a Design Space for Non-Flat Interactive Displays. In Workshop: Beyond Flat Displays, In conjunction with ITS 2012.

[4] Rümelin, S., Brudy, F. and Butz, A. Up And Down And Along: How We Interact With Curvature. In Workshop: Displays Take New Shape: An Agenda for Interactive Surfaces, In conjunction with CHI 2013.

[5] Weiss, M., Voelker, S., Sutter, C. and Borchers, J. BendDesk: dragging across the curve. In Proc. ITS 2012. ACM Press (2010), 1-10.

[6] Wiethoff, A. and Gehring, S. Designing interaction with media façades: a case study. In Proc. DIS 2012. ACM Press (2012), 308-317.

[7] Wimmer, R., Hennecke, F., Schulz, F., Boring, S., Butz, A. and Hußmann, $H$. Curve: revisiting the digital desk. In Proc. NordiCHI 2010. ACM Press (2010), 561570 . 